\title{
Increasing and Decreasing with Fuzzy Time Series
}

\author{
Ming-Tao Chou ${ }^{* 1}$ Hsuan-Shih Lee ${ }^{2}$ \\ ${ }^{1}$ Depatrtment of Aviation and Martime Managemnet, Chang Jung Christian University \\ ${ }^{2}$ Department of Shipping and Transportation Managemnet, National Taiwan Ocean University
}

\begin{abstract}
There is a significant problem associated with the fuzzy time series. That is a strict increasing and decreasing case. Under the discussion case, fuzzy time series model arise a continuous increasing/decreasing forecasting value. From the illustrative example, we can see that our definition not only define the trend of the fuzzy numbers that represent the linguistic values of the linguistic variable more appropriately, but also can make the fuzzy time series reasonable.
\end{abstract}

Keywords: Fuzzy sets; Fuzzy forecasting.

\section{Introduction}

The main purpose of this article is to present a mathematical skill to construct a fuzzy time series model of a system. There has been a considerable of studies [1, 3-5] on fuzzy time series where fuzzy [6-9] implications are used to express forecasting rules. Many kinds of fuzzy time series model have been developed since Lee and Chou's [5] paper.

In this article, we present a new definition for forecasting with fuzzy time series based on Lee and Chou's method. The historical data of the Port of Kaohsiung used by Lee and Chou's method [5] are adopted to justify that our method is as reasonable as Lee and Chou's method but more complete than original method. In forecasting Port of Kaohsiung throught, there is a significant problem associated with the fuzzy time series. That is the historical data shown strict increasing and decreasing situation. According to Lee and Chou's method [5], we add a definition to conquer the attribution, based on the definition the fuzzy time series is more complete.

The remainder of the article is structured as follows. Section 2, presents the fuzzy time series definition. Section 3, we present a new definition for forecasting with fuzzy time series based on Lee and Chou's method, our method is also evaluated in section 4. Finally, the conclusions are made in section 5.

\section{Fuzzy sets and Fuzzy time series}

In 1965, Zadeh [6] introduced some basic concepts and terminology of "Fuzzy Sets". It makes the start of a scientific revolution related to uncertainty, and the effects of which are still being explored. In 1978, Dubios and Prade [2] defined the fuzzy number as a fuzzy subset of the real line. In 1975, Zadeh [9] introduced fuzzy arithmetic theory and its application.

\section{Definition 2.1}

A fuzzy number in real line $\mathfrak{R}$ is a fuzzy subset of $\mathfrak{R}$ that is normal and convex.

The fuzzy time series was first presented by Song and Chissom [3, 4]. There are a number of calculation methods to determine the fuzzy relation. Different methods will yield different results $[3,5,10]$. In their papers, they followed Mamdani's method [3] to determine the relations. The main difference between the fuzzy time series and traditional time series is that the values of the former are fuzzy sets while the values of the latter are real historical numbers. The definitions of fuzzy time series are reviewed as follows.

Definition 2.2 [3, 4]

Let $Y(t)(t=\ldots, 0,1,2, \ldots)$, a subset of $\Re$, be the universe of discourse on which fuzzy sets $f_{i}(t)(i=1,2, \ldots)$ are defined and $F(t)$ is the collection of $f_{i}(t)(i=1,2, \ldots)$. Then ${ }_{F(t)}$ is called a fuzzy time series on ${ }^{Y}(t)(t=\ldots, 0,1,2, \ldots)$.

$F(t)$ can be understood as a linguistic variable and $f_{i}(t)(i=1,2, \ldots)$ as the possible linguistic values of $F(t)$. Because at different times, the values of $F(t)$ can be different, $F(t)$ is a function of time $t$. Also, since the universes of discourse can be different at different times, ${ }^{Y}(t)$ is used for the universe at time $t$.

\footnotetext{
${ }^{*}$ Corresponding author. Tel.:886-6-278-5123 ext.2262; fax:886-6-278-5056.

E-mail address: mtchou@mail.cju.edu.tw
} 
Definition 2.3 [3, 4]

Let $I$ and $J$ be indices sets for $F(t-1)$ and $F(t)$ respectively. If for any $_{f(t) \in F(t)}$ where $j \in J$, there exists $f(t-1) \in F(t-1)$ where $i \in I$ such that there exists a fuzzy relation $R_{i j}(t, t-1)$ and $f_{j}(t)=f_{i}(t-1) \circ R_{i}(t, t-1)$ where ' $\circ$ ' is the max-min composition, then $F(t)$ is said to be caused by $F_{(t-1)}$ only. Denote this as $f_{i}(t-1) \rightarrow f_{i}(t)$ or equivalently $F(t-1) \rightarrow F(t)$.

Definition $2.4[3,4]$

If for any $f(t) \in F(t)$ where $j \in J$, there exists $f_{i}(t-1) \in F(t-1)$ where $i \in I$ and a fuzzy relation $R_{i j}(t, t-1)$ such that $f_{j}(t)=f_{i}(t-1) \circ R_{i j}(t, t-1)$, let $R(t, t-1)=\bigcup, R_{i}(t, t-1)$ where' $\bigcup$ 'is the union operator. Then $R(t, t-1)$ is called the fuzzy relation between $F(t)$ and $F_{(t-1)}$ and define this as the following fuzzy relational equation: $F(t)=F(t-1) \circ R(t, t-1)$.

Definition 2.5 $[3,4]$

Suppose $_{R_{1}(t, t-1)=\bigcup R_{v}^{\prime}(t, t-1)}$ and $R_{2}(t, t-1)=\bigcup_{R_{v}^{2}(t, t-1)}$ ar e two fuzzy relations between $F(t)$ and $F_{(t-1)}$. If for any $f_{j}(t) \in F(t)$ where $j \in J$, there exists $f_{i}(t-1) \in F(t-1) \quad$ where $i \in I$ and fuzzy relations $\quad R_{i j}^{1}(t, t-1) \quad$ and $R_{i j}^{2}(t, t-1) \quad$ such that $f_{j}(t)=f_{i}(t-1) \circ R_{i j}^{\prime}(t, t-1)$ and $f_{j}(t)=f_{i}(t-1) \circ R_{i j}^{2}(t, t-1)$, then define $R_{1}(t, t-1)=R_{2}(t, t-1)$.

Definition 2.6 [3, 4]

Suppose $_{F(t)}$ is caused by $F(t-1)$ only or by

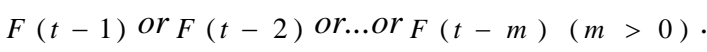

This relation can be expressed as the following fuzzy relational equation:

$F(t)=F(t-1) \circ R(t, t-m)$

or

$F(t)=(F(t-1) \cup F(t-2) \cup \ldots \cup F(t-m))$

$\circ R_{0}(t, t-m)$

Then equation 2-1 or 2-2 is called the first-order model of $F(t)$.

Definition 2.7 [3, 4]

Suppose $_{F(t)}$ is caused by $F(t-1), F(t-2), \ldots$, and $F(t-m)(m>0)$ simultaneously. This relation can be expressed as the following fuzzy relation equation:

$F(t)=(F(t-1) \times F(t-2) \times \ldots \times F(t-m)) \circ R_{a}(t, t-m)$

Then equation 2-3 is called the mth order model of $F(t)$.

To simplify the assumption, Chen assumed $_{F(t)}$ is singleton. That is, $F(t)$ is a fuzzy set not a set of fuzzy sets. The simplified fuzzy time series is defined as follows.

\section{Definition 2.8 [5]}

$F(t)$ is a fuzzy time series if $F_{(t)}$ is a fuzzy set. The transition is denoted as $F(t-1) \rightarrow F(t)$.

\section{Definition 2.9 [5]}

The universe of discourse $U=\left[D_{L}, D_{U}\right]$ is defined such that $D_{L}=D_{\min }-\frac{s}{\sqrt{n}} t_{\alpha}(n)$ and $D_{U}=D_{\max }+\frac{s}{\sqrt{n}} t_{\alpha}(n)$ when $n \leq 30$ or $D_{L}=D_{\min }-\frac{s}{\sqrt{n}} z_{\alpha}$ and $D_{U}=D_{\max }+\frac{s}{\sqrt{n}} z_{\alpha}$ when $n>30$, where $t_{\alpha}(n)$ is $100(1-\alpha)$ percentile of the $t$ distribution with $n$ degrees of freedom and $z_{\alpha}$ is the $100(1-\alpha)$ percentile for the standard normal distribution, that is, if $Z$ is $N(0,1)$ distribution then $P\left(Z \geq z_{\alpha}\right)=\alpha$.

Definition 2.10 [5]

Assume there are $m$ linguistic values under consideration. Let $A_{i}$ be the fuzzy number that represents the ith linguistic value of the linguistic variable where $1 \leq i \leq m$. The support of $A_{i}$ is defined to be

$\left\{\begin{array}{l}{\left[D_{L}+(i-1) \frac{D_{U}-D_{L}}{m}, D_{L}+\frac{i\left(D_{U}-D_{L}\right)}{m}\right), 1 \leq i \leq m-1} \\ {\left[D_{L}+(i-1) \frac{D_{U}-D_{L}}{m}, D_{L}+\frac{i\left(D_{U}-D_{L}\right)}{m}\right], i=m .}\end{array}\right.$

\section{A new definition for forecasting with fuzzy time series}

In the following, we propose a new definition to forecast by fuzzy time series. Generally, our method is a modification of the method proposed by Lee and Chou [5]. We add a new definition is more complete than Lee and Chou method due to the fact that we define stability concept of the fuzzy time series that represent the increasing and the decreasing case more appropriately.

In this article, we apply difference test to understand whether information are in stable state. If the information location is in unstable state, do the second difference to divide and continue assaying, recursion until the information are in stable state again. Let $d(t)-d(t-1)$ is difference value, $d(t)$ is $t^{\text {th }}$ historical data, $d(t-1)$ is $(t-1)^{\text {th }}$ historical data respectively.

\section{Definition 3.1}

For test $H_{0}$ : non fuzzytrend against $H_{1}$ : fuzzytrend,

the test given by

$P(F(t)>F(t-1)) \geq 1-\lambda$,

$P(F(t-1)>F(t)) \geq 1-\lambda$,

$P(F(t)>F(t-1))=P(F(t-1)>F(t))=0.5$,

$0<\lambda<0.5$.

Where Critical region: 
$C^{*}=\left\{C \mid C_{2}^{k}+C_{2}^{n-k}>C_{\lambda}=C_{2}^{n} \times(1-\lambda)\right\}, \alpha$ is significantly level, initial value vale is 0.2 or 0.3 . This initial value is stricter if the value is near 0.5. Let $k$ is the number of differential.

Our forecasting algorithm based on fuzzy time series works as follows:

\section{Step 1:}

Let $d(t)$ be the history data under consideration and $F(t)$ be the fuzzy time series. Difference test to understand whether information are in stable state according to definition 3.1., recursion until the information are in stable state again. Where critical region $C^{*}=\left\{C \mid C_{2}^{k}+C_{2}^{n-k}>C_{\lambda}=C_{2}^{n} \times(1-\lambda)\right\}$.

Determine the universe of discourse $U=\left[D_{L}, D_{U}\right]$ according to definition 2.9.

Step 3:

Following the definition of 2.10 , define $A_{i}$ by letting its membership function

$u_{A_{i}}(x)=\left\{\begin{array}{l}1 \text { for } x \in\left[D_{L}+(i-1) \frac{D_{U}-D_{L}}{m}, D_{L}+\frac{i\left(D_{U}-D_{L}\right)}{m}\right) \text { wherel } \leq i \leq m-1 \\ 1 \text { for } x \in\left[D_{L}+(i-1) \frac{D_{U}-D_{L}}{m}, D_{L}+\frac{i\left(D_{U}-D_{L}\right)}{m}\right] \text { where } i=m \\ 0 \text { otherwise. }\end{array}\right.$

Step 4:

Then $F(t)=A_{i}$ if $d(t) \in \operatorname{supp}\left(A_{i}\right)$, where $\operatorname{supp}(\cdot)$ denote the support.

Step 5:

Derive the transition rule from period $t-1$ to $t$ and denote it as $F(t-1) \rightarrow F(t)$. Aggregate all transition rules. Let the set of rules be $R=\left\{r_{i} \mid r_{i}: P_{i} \rightarrow Q_{i}\right\}$.

Step 6:

The value of $d(t)$ can be predicted by fuzzy time series $F(t)$ as follows:

Let $T(t)=\left\{r_{j} \mid d(t) \in \operatorname{supp}\left(P_{j}\right)\right.$, where $\left.r_{j} \in R\right\}$ be the set of rules fired by $d(t)$, where $\operatorname{supp}\left(P_{j}\right)$ is the support of $P_{j}$. Let $\overline{\operatorname{supp}\left(P_{j}\right)}$ be the median of $\operatorname{supp}\left(P_{j}\right)$. The predicted value for $d(t)$ is

$$
\sum_{r_{j} \in T(t-1)} \frac{\overline{\operatorname{supp}\left(Q_{j}\right)}}{|T(t-1)|} \text {. }
$$

\section{Forecasting history data with our method}

We verify our method by forecasting the container throught by harbors in Taiwan area from 1973 to 2003 as shown in Table 1.

\section{Step 1:}

Let $d(t)$ be the history data under consideration and $F(t)$ be the fuzzy time series. Difference test to understand whether information are in stable state according to definition 3.1., recursion until the information are in stable state again.

Difference one $=$

$C^{*}=\left\{C \mid C_{2}^{31-1} \times(1-0.2)\right\}=372$.

$\Rightarrow$ do not reject $H_{0}$

Difference two $=$

$C^{\prime \prime}=\left\{C \mid C=C_{2}^{17}+C_{2}^{13}\right\}=214<\left\{C^{\prime} \mid C=C_{2}^{30} *(1-0.2)\right\}=348$

$\Rightarrow$ do not reject $H_{0}$

\section{Step 2:}

Determine the universe of discourse $U=\left[D_{L}, D_{U}\right]$ :

By definition 2.9, the discourse $U=\left[D_{L}, D_{U}\right]$ from

Table 1, we have $D_{\min }=-83,048, D_{\max }=1,180,920$, $s=311,069.74$, and $n=30$. Let $\alpha=0.05$. Because $\mathrm{n}$ is less than 30, we use Student-t distribution with 30 degrees of freedom as a substitute for normal distribution. That is $U=[-179,430,1,277,302]$.

\section{Step 3:}

Assume the following linguistic values are under consideration: extremely few, very few, few, some, many, very many, and extremely many. Following Definition 2.10, the supports of fuzzy numbers that represent the linguistic values are given by

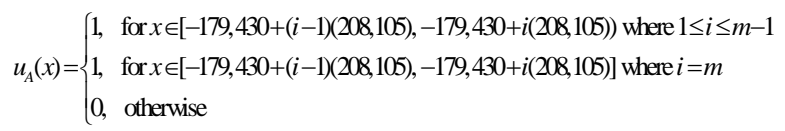

such that $A_{1}$ ='extremely few', $A_{2}$ ='very few', $A_{3}$ ='few', $A_{4}$ ='some', $A_{5}$ ='many', $A_{6}=$ 'very many', and $A_{7}=$ 'extremely many'. Thus, we have $\operatorname{supp}\left(A_{1}\right)=[-179,430,208,675)$

$\operatorname{supp}\left(A_{2}\right)=[28,675,236,780)$

$\operatorname{supp}\left(A_{3}\right)=[236,780,444,885)$

$\operatorname{supp}\left(A_{4}\right)=[444,885,652,990)$

$\operatorname{supp}\left(A_{5}\right)=[652,990,861,095)$

$\operatorname{supp}\left(A_{6}\right)=[861,095,1,069,200)$

$\operatorname{supp}\left(A_{7}\right)=[1,069,200,1,277,302]$

Step 4:

The fuzzy time series $F(t)$ is given by $F(t)=A_{i}$ when $d(t) \in \operatorname{supp}\left(A_{i}\right)$. Therefore, $F(1974)=A_{1}, F(1975)=A_{2}, F(1976)=A_{2}$, $F(1977)=A_{2}, \ldots$, and $F\left(\begin{array}{llll}2 & 0 & 0 & 3\end{array}\right)=A_{4}$

The correspondences between actual enrollments and fuzzy enrollments are shown in Table 1.

\section{Step 5:}

The transition rules are derived from Table 1. For example, $F(1974) \rightarrow F(1975)$ is $A_{1} \rightarrow A_{2}$. All transition rules obtained from Table 1 are shown in Table 2.

Step 6:

We are going to forecast based on Table 2. The forecasting results from 1974 to 2003 are shown in Table 3. The forecasting process of year 2004 are illustrated in the following. The same process can be applied to other years. 
Year 2004 To predict the enrollment of 2004, let $\mathrm{t}=2004$. Since $d^{\prime}(t-1)=d^{\prime}(2003)=486,119 \in \operatorname{supp}\left(A_{4}\right)$, $T(t-1)=T(2003)=\left\{r_{11}: A_{4} \rightarrow A_{2}, r_{12}: A_{4} \rightarrow A_{3}, r_{13}: A_{4} \rightarrow A_{4}\right\}$. Thus the forecasted value for difference one $d^{\prime}(t)=d^{\prime}(1972)$ is

$\sum_{r_{j} \in T(2003)} \frac{\overline{\operatorname{supp}\left(Q_{j}\right)}}{|T(2003)|}=\frac{\overline{\operatorname{supp}\left(A_{2}\right)+\operatorname{supp}\left(A_{3}\right)+\operatorname{supp}\left(A_{4}\right)}}{3} \approx 340,832$.

Year 2004 of container throught by harbors in Taiwan area is $d(2003)+d^{\prime}(2003)=12,094,753+340,832=12,435,585$.

Table 1. Fuzzy historical TEUs ${ }^{1}$

\begin{tabular}{|c|c|c|c|c|}
\hline $\begin{array}{c}\text { Year } \\
(\mathrm{t})\end{array}$ & $\begin{array}{c}\text { TEUs } \\
\mathrm{d}(\mathrm{t})\end{array}$ & $\begin{array}{c}\text { Difference one } \\
d^{\prime}(t)\end{array}$ & $\begin{array}{l}\text { Difference } \\
\text { two d', (t) }\end{array}$ & $\begin{array}{l}\text { Fuzzy } \\
\text { TEUs }\end{array}$ \\
\hline 1973 & 370,372 & & & \\
\hline 1974 & 391,354 & 20,982 & & $\mathrm{~A}_{1}$ \\
\hline 1975 & 473,353 & 81,999 & 61,017 & $\mathrm{~A}_{2}$ \\
\hline 1976 & 661,866 & 188,513 & 106,514 & $\mathrm{~A}_{2}$ \\
\hline 1977 & 755,258 & 93,392 & $-95,121$ & $\mathrm{~A}_{2}$ \\
\hline 1978 & $1,062,561$ & 307,303 & 213,911 & $\mathrm{~A}_{3}$ \\
\hline 1979 & $1,374,392$ & 311,831 & 4,528 & $A_{3}$ \\
\hline 1980 & $1,644,376$ & 269,984 & $-41,847$ & $A_{3}$ \\
\hline 1981 & $1,787,758$ & 143,382 & $-126,602$ & $\mathrm{~A}_{2}$ \\
\hline 1982 & $1,902,264$ & 114,506 & $-28,876$ & $\mathrm{~A}_{2}$ \\
\hline 1983 & $2,429,310$ & 527,046 & 412,540 & $\mathrm{~A}_{4}$ \\
\hline 1984 & $3,026,846$ & 597,536 & 70,490 & $\mathrm{~A}_{4}$ \\
\hline 1985 & $3,075,151$ & 48,305 & $-549,231$ & $\mathrm{~A}_{2}$ \\
\hline 1986 & $4,104,953$ & $1,029,802$ & 981,497 & $\mathrm{~A}_{6}$ \\
\hline 1987 & $4,772,339$ & 667,386 & $-362,416$ & As \\
\hline 1988 & $4,941,022$ & 168,683 & $-498,703$ & $\mathrm{~A}_{2}$ \\
\hline 1989 & $5,263,091$ & 322,069 & 153,386 & $\mathrm{~A}_{3}$ \\
\hline 1990 & $5,463,566$ & 200,475 & $-121,594$ & $\mathrm{~A}_{2}$ \\
\hline 1991 & $6,129,667$ & 666,101 & 465,626 & As \\
\hline 1992 & $6,178,872$ & 49,205 & $-616,896$ & $\mathrm{~A}_{2}$ \\
\hline 1993 & $6,824,973$ & 646,101 & 596,896 & $\mathrm{~A}_{4}$ \\
\hline 1994 & $7,307,304$ & 482,331 & $-163,770$ & $\mathrm{~A}_{4}$ \\
\hline 1995 & $7,665,178$ & 357,874 & $-124,457$ & $\mathrm{~A}_{3}$ \\
\hline 1996 & $7,866,995$ & 201,817 & $-156,057$ & $\mathrm{~A}_{2}$ \\
\hline 1997 & $8,520,199$ & 653,204 & 451,387 & As \\
\hline 1998 & $8,858,211$ & 338,012 & $-315,192$ & $\mathrm{~A}_{3}$ \\
\hline 1999 & $9,757,648$ & 899,437 & 561,425 & $A_{6}$ \\
\hline 2000 & $10,510,762$ & 753,114 & $-146,323$ & As \\
\hline 2001 & $10,427,714$ & $-83,048$ & $-836,162$ & $\mathrm{~A}_{1}$ \\
\hline 2002 & $11,608,634$ & $1,180,920$ & $1,263,968$ & $\mathrm{~A}_{7}$ \\
\hline 2003 & $12,094,753$ & 486,119 & $-694,801$ & $\mathrm{~A}_{4}$ \\
\hline
\end{tabular}

Table 2. Fuzzy transitions derived from Table 1.

\begin{tabular}{lll}
\hline$r_{1}: A_{1} \rightarrow A_{2}$ & $r_{7}: A_{2} \rightarrow A_{6}$ & $r_{13}: A_{4} \rightarrow A_{4}$ \\
$r_{2}: A_{1} \rightarrow A_{7}$ & $r_{8}: A_{3} \rightarrow A_{2}$ & $r_{14}: A_{5} \rightarrow A_{1}$ \\
$r_{3}: A_{2} \rightarrow A_{2}$ & $r_{9}: A_{3} \rightarrow A_{3}$ & $r_{15}: A_{5} \rightarrow A_{2}$ \\
$r_{4}: A_{2} \rightarrow A_{3}$ & $r_{10}: A_{3} \rightarrow A_{6}$ & $r_{16}: A_{5} \rightarrow A_{3}$ \\
$r_{5}: A_{2} \rightarrow A_{4}$ & $r_{11}: A_{4} \rightarrow A_{2}$ & $r_{17}: A_{6} \rightarrow A_{5}$ \\
$r_{6}: A_{2} \rightarrow A_{5}$ & $r_{12}: A_{4} \rightarrow A_{3}$ & $r_{18}: A_{7} \rightarrow A_{4}$ \\
\hline
\end{tabular}

\section{Conclusions}

In this article, we add a useful definition to improve original method [4] to forecast the strict increasing and decreasing case based on fuzzy time series. From the illustrative example, we can see that our definition not

\footnotetext{
${ }^{1}$ TEUs $=20$-foot equivalent containers
}

only define the trend of the fuzzy numbers that represent the linguistic values of the linguistic variable more appropriately, but also can make the fuzzy time series reasonable. In summary, our method is as accurate as Lee and Chou method [4] but more reasonable in forecasting the strict increasing and decreasing case.

\section{Acknowledgement}

This research work was supported by the National Science Council of the Republic of China under grant No. NSC 94-2211-E-309-002.

\section{References}

[1] S. M. Chen, "Forecasting enrollment based on fuzzy time series," Fuzzy sets and Systems, 81, pp.311-319, 1996.

[2] D. Dubios, and H. Prade, "Operations on fuzzy numbers,” Int. J. System Science, 9, 1-6, 1978.

[3] Q. Song and B.S. Chissom, "Forecasting enrollment with fuzzy time series-part I," Fuzzy sets and Systems, 54, pp.1-9, 1993.

[4] Q. Song and B.S. Chissom, "Forecasting enrollment with fuzzy time series-part II," Fuzzy sets and Systems, 54, pp.267-277, 1994.

[5] Lee, H.-S. and Chou, M.-T., "Fuzzy forecast based on fuzzy time series," International Journal of Computer Mathematic, 81 (7), pp.781-789, 2004.

[6] L.A. Zadeh, "Fuzzy set," Information and Control, 8, pp.338-353, 1965.

[7] L.A. Zadeh, "Outline of a new approach to the analysis of complex system and decision process," IEEE Tran. System Man Cybernet., 3, pp.28-44, 1973.

[8] L.A. Zadeh, "Similarity relations and fuzzy orderings," Information Sciences, 3, pp.177-200, 1971.

[9] L.A. Zadeh, "The concept of a linguistic variable and its application to approximate reasoning, Part 1-3," Inform. Sci., 8, pp.199-249, $1975 ; 8$ pp.301-357, 1975; 9, pp.43-80, 1975.

[10] M. Sugeno and K. Tanaka, "Successive identification of a fuzzy model and its applications to prediction of a complex system," Fuzzy Sets and Systems, 42, pp. 315-334, 1991. 